\title{
Mars Sample Return Campaign Concept Architecture
}

A White Paper for the Planetary Science and Astrobiology Decadal Survey 2023-2032

Primary Author: Brian K. Muirhead, brian.k.muirhead@jpl.nasa.gov, phone: (818) 687-7003. Jet Propulsion Laboratory, California Institute of Technology 4800 Oak Grove Drive, Pasadena, CA 91109

\section{Co-Authors:}

Austin K. Nicholas ${ }^{\text {a }}$, austin.k.nicholas@jpl.nasa.gov

Chad Edwards ${ }^{\mathbf{a}}$, charles.d.edwards@jpl.nasa.gov

Jeffrey Umland ${ }^{\mathbf{a}}$, jwumland@jpl.nasa.gov

Sanjay Vijendran ${ }^{\mathbf{b}}$, vijendran@esa.int

Richard Zurek ${ }^{\mathrm{a}}$, richard.w.zurek@jpl.nasa.gov

a Jet Propulsion Laboratory, California Institute of Technology, Pasadena, CA

${ }^{b}$ European Space Agency, Noordwijk, The Netherlands

\section{Executive Summary}

The Planetary Science and Astrobiology Decadal Survey 2023-2032 (hereafter the Survey) has been asked by NASA to assess whether the Agency's "plans for Mars Sample Return (MSR) play an appropriate role in the research strategy for the next decade". This white paper provides an overview of the campaign concept architecture.

MSR is currently envisioned to be a campaign of three flight elements and one ground element. The Mars 2020 mission with its Perserverance caching rover is the first element and is on schedule to launch this July. This paper describes the other two flight elements envisioned for MSR, namely a Sample Return Lander and Earth Return Orbiter, that are being jointly studied by NASA and the European Space Agency (ESA). Overall objectives and mission options will be described, including the campaign architecture's constraints and notional timelines. The paper will highlight architecture-level trade studies and their current status as part of the reference architecture proceeding toward possible project approval to enter Phase A.

The MSR campaign architecture is well understood, with major progress on the technical trades and key technology developments. NASA and ESA are working together well in an effective partnership, with roles and responsibilities in place and operating.

With this study phase at a very mature state, preparations are being made for a MSR Campaign Concept Review in late summer 2020, as part of the process by which NASA will decide whether MSR is ready to proceed into formulation. ESA has already received approval, at their ministerial level, to proceed forward with their part of the program.

Keywords (acronyms): Mars Sample Return (MSR), Sample Retrieval Lander (SRL), Sample Fetch Rover (SFR), Mars Ascent Vehicle (MAV), Orbiting Sample (OS) container, Capture/Containment and Return System (CCRS), Earth Return Orbiter (ERO), Earth Entry System (EES)

Acknowledgment: Part of this work was carried out at the Jet Propulsion Laboratory, California Institute of Technology, under contract with the National Aeronautics and Space Administration. 


\section{Introduction}

The previous planetary decadal survey report [1] Visions and Voyages for Planetary Science in the Decade 2013-2022 (hereafter V\&V2011) recommended, as its highest-priority flagship mission for the decade, the Mars Astrobiology Explorer-Cacher (MAX-C) to begin a NASAESA Mars Sample Return campaign. In that scenario, MAX-C was a caching rover to be jointly landed with the ExoMars rover. Due to the complexity and cost of landing two substantial, independently designed rovers with a single landing system, these two rovers were ultimately separated, and MAX-C became a single rover, the Mars 2020 rover, with a major emphasis on in situ science, but also with the capability to cache samples, whose means and schedule of return were left for future definition.

That changed with a presentation from the NASA Associate Administrator, Dr. Thomas Zurbuchen, to the National Academies on August 28, 2017 [2]. Mindful of V\&V2011's recommendation that such an ambitious enterprise must also involve ESA participation, NASA and ESA officially began a new collaboration with a joint NASA/ESA Statement of Intent (signed in Berlin on 4/26/18). Earlier scenarios of this architecture and its concepts for an MSR campaign have been presented at various international forums including the Second International Mars Sample Return Conference in April 2018, in Berlin and the International Astronautics Congress in Oct. 2018 in Bremen.

As recognized in $V \& V 2011$, the Mars science community continues to see a sample return mission as the logical next step in Mars exploration. The international MSR Objectives and Samples Team (iMOST) re-evaluated and updated the sample-related science and engineering objectives of a MSR campaign [3]. As described in the companion white paper [4], that team found that the samples to be prepared by the Mars 2020 rover at its Jezero Crater landing site would address compelling science questions of habitability (pre-biotic chemistry and possible biology) and planetary evolution (of atmospheres, surface and crusts), not only for Mars, but for the Solar System.

$V \& V 2011$ (p. 270) fully recognized that their committee had recommended a plan for their coming decade that "also had signficant budget implications for one or even two decades beyond". They also recognized (p. 276) there had to be "significant technology investments in the Mars Sample Retrieval Lander [and] Mars Sample Return Orbiter..." to mature key technologies and to reduce costs and risks. In the years since, NASA and ESA have made significant investments that have yielded the overall campaign architecture, mission concepts, and maturation of key technologies described here.

\section{Planning for a Mars Sample Return Campaign}

\subsection{MSR Conceptual Architecture}

MSR is currently envisioned to be made up of three flight elements and one ground element. This is the same (with slightly different nomenclature) as the mission architecture evaluated in $V \& V 2011:$ 1) A caching rover, now the Mars 2020 Perseverance, 2) a Sample Retreival Lander (SRL), and 3) an Earth Return Orbiter (ERO).

The ground element would be a Mars Returned Sample Handling (MRSH) facility, with international governance, that will be discussed in a subsequent white paper.

Mars 2020 Perserverance is responsible for sample selection, context characterization, acquisition and caching. Ready for launch in July 2020, it will land in February 2021 in Jezero Crater, a site selected after 4 years of study and discussion of extensive remote sensing data.

The next flight elements are the SRL which would carry: a) a Sample Fetch Rover (SFR) to retrieve the cached samples for placement in the Orbiting Sample (OS) container, and b) a Mars Ascent Vehicle (MAV) to launch the OS into Mars orbit. A sample transfer arm (STA) will 
robtotically transfer the sample tubes from the fetch rover into the OS. The ERO would carry the Capture/Containment and Return System (CCRS), which would capture and contain the OS breaking the chain of back contamination. The OS is then placed into an Earth Entry System (EES) which the ERO releases near Earth for eventual return to the surface.

\subsection{Functional Objectives}

The campaign functional objectives for the next flight elements in a potential NASA/ESA MSR joint campaign are:

- Retrieve and return to Earth a scientifically selected set of Mars samples for investigation in terrestrial laboratories, thereby achieving a set of sample-related scientific objectives addressing life, geologic environments, geochronology, volatiles, environmental hazards, and InSitu Resource Utilization (ISRU).

- Ensure the scientific integrity of the returned samples through contamination control (including round-trip Earth contamination and sample-to-sample crosscontamination) and control of environments experienced by the samples after acquisition.

- Ensure compliance with planetary protection (PP) requirements associated with the return of Mars samples to Earth's biosphere.

\subsection{NASA/ESA Roles in MSR}

The joint NASA/ESA Statement of Intent has divided the project responsibilities as follows: NASA would have overall campaign responsibility and would provide the SRL and the MAV, the CCRS including the Earth Entry System (EES), which the ERO would carry. The ERO itself and the SFR would be provided by ESA. ESA would also provide the Sample Transfer Arm on the NASA provided SRL.

\subsection{Operations Timeline Concept for Arrival}

The latest conceptual MSR campaign timeline proposes a 2026 launch which could return samples as soon as 2031. [3] The notional timeline has four essential features:

- After an extended cruise, SRL would arrive at the beginning of northern spring at a time of seasonally high atmospheric pressure which would improve entry/descent/landing (EDL) performance and thus mass margin.

- Arrival at that time followed by launch of the MAV before Northern Fall enables a solar-power-only mission during the Mars seasons of lowest atmospheric dust opacity.

- After a later launch than the SRL but on a shorter cruise, the ERO has time to sprial down into low Mars orbit, enabling telecom coverage of SRL EDL and full relay support for surface operations for both SRL and SFR.

- SRL and ERO trajectories are feasible and fit into available launch vehicle options.

\subsection{Backward Planetary Protection}

The return of samples from Mars to Earth requires a Planetary Protection Categorization $\mathrm{V}$, with restricted return, which is a requirement to prevent uncontained or unsterilized material from Mars from being released into Earth's biosphere. This drives the design of MSR systems to return the Mars sample tubes in the OS to Earth, while containing, immobilizing and/or sterilizing any other Mars material that the MSR elements may have contacted.

The methodologies used to achieve this are referred to as "Break-the-Chain," (of contact with Mars). The key elements of this "Breakthe-Chain" strategy include [5]:

- Establishing design, test and verification goals and requirements;

- Use of various modeling tools, including fault trees to analyze performance and failure modes as part of the design process;

- Analysis of dust transport mechanisms and mitigations;

- Containment techniques;

- Sterilization Methodologies and modalities;

- Model validation testing. 


\subsection{Key Trade Studies and Systems Engineering}

The systems engineering team has developed trade studies while assessing options to achieve the most robust overall architecture for the campaign lifecycle as judged by:

- Campaign-level mission success;

- Number of sample tubes returned to Earth;

- Development and operational risk;

- Implementation approach;

- Cost.

Key metrics being used within the trade space and between the elements are:

- Mass and power, and associated margins;

- Schedule margin (development);

- Timeline margin, including overall orbital and surface operations timeline;

- Planetary protection metrics (e.g. dust and particle reduction factors, containment element performance);

- EDL performance and margins, including landing accuracy, for both SRL and EES;

- MAV and ERO rendezvous and capture performance and margins.

\section{Lander Concepts Under Study}

The multi-center (JPL, MSFC, LaRC, and ARC) and international team working on the MSR SRL have studied two lander concepts: a Propulsive Platform Lander (PPL, Fig. 1) and a Sky-Crane Delivered Lander (SDL, Fig. 2). The SRL must land on Mars, deploy the SFR, and maintain the lander and the MAV (provided by MSFC) within safe operating conditions, including temperatures, while the SFR retrieves the M2020 sample tubes.

If still operating, Perserverance may also deliver sample tubes to the SRL. Once either or both of the rovers arrive with sample tubes, the Sample Transfer Arm (STA) transfers the tubes into the OS in the MAV payload area. The SRL then prepares to launch the MAV (heating it to the operational temperature and erecting it).

The EDL technology proposed for both SRL options is mostly common and is based on

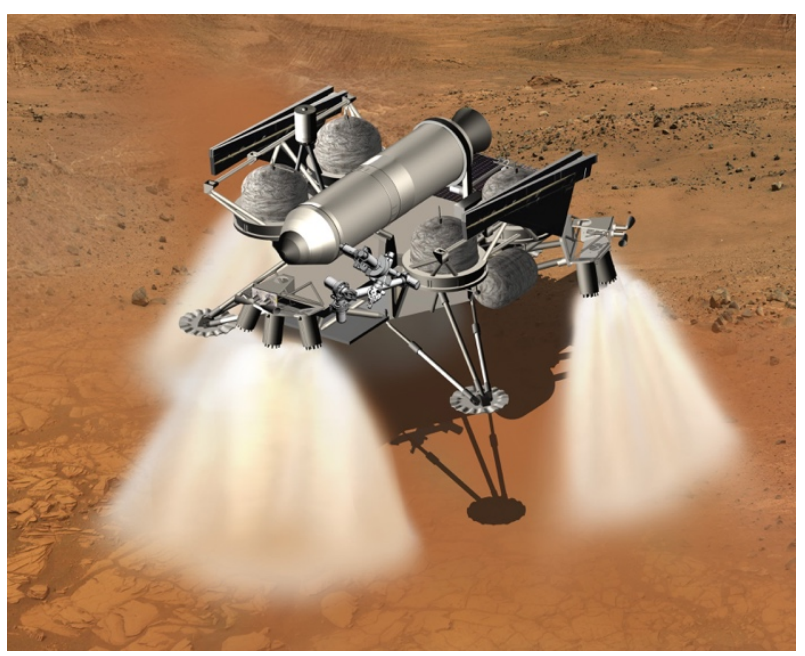

Figure 1: Propulsive Platform Lander Concept

Mars Science Laboratory and Mars 2020 upgrades, including terrain relative navigation (TRN). SRL is using TRN to enable an extended divert capability that will allow landing within 20 meters of the desired target.

\section{Figure 2: Skycrane Delivered Lander Concept}

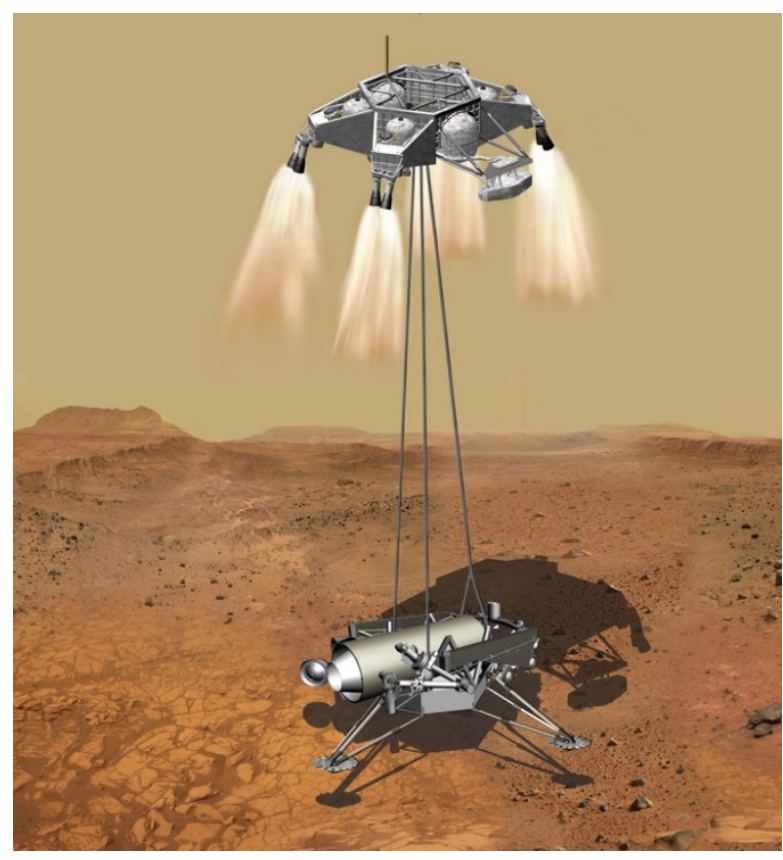

The major lander design drivers are accommodation of both the MAV (400-500 kg with margin) and its launch system, and the SFR (200-230 kg with margin) and its egress system (provided by NASA). 
The solar power system and thermal design are being developed for the worst-case environments, which are relatively benign at the northern hemisphere Jezero site given arrival in Mars spring and launch of the MAV before fall.

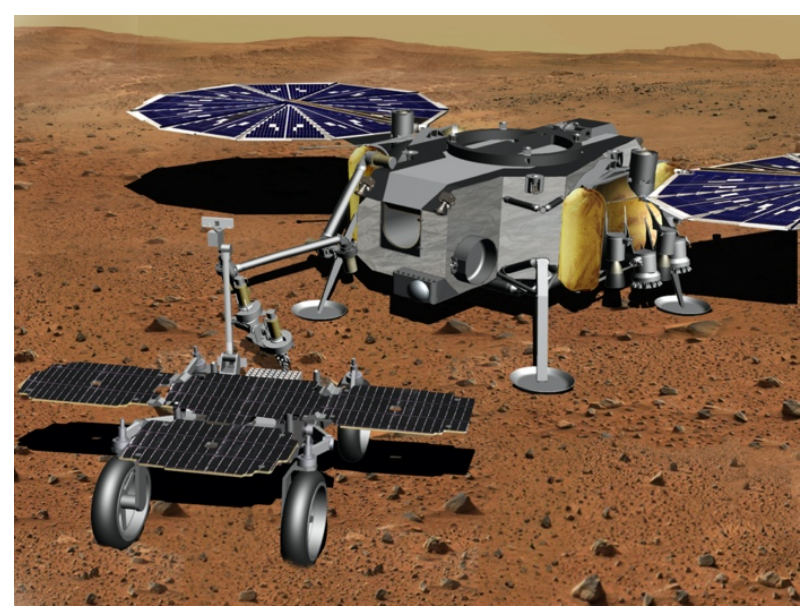

Figure 3: Sample Transfer Arm transferring sample tubes from SFR to MAV OS on SRL.

For cruise, the spinning cruise stage and backshell are based on MSL designs. Both concepts utilize a 4.7-m spherical heatshield (different from sphere-cone used on MSL and M2020) to improve EDL performance and gain greater volume for packaging the lander.

The PPL concept employs a propulsion system similar to Viking, Phoenix or Insight, with descent and landing thrusters as part of the platform itself. The current strategy is to use six M2020 engines with the standard nozzle and four engines with showerhead-type nozzles for terminal descent, to reduce plume interaction with the surface. This concept provides larger configuration and packaging flexibility/margin (in both volume and mass).

The SDL concept provides a softer landing with less plume/ground interactions due to the Skycrane technology. However, the need to accommodate the MAV and SFR, as well as additional propellant for extended divert, results in significant mass margin and packaging volume problems for the SDL.

Based on results from the architecture closure process, the PPL was selected as the reference baseline.

\section{Orbiting Sample (OS) Container, MAV Payload Assembly (MPA) and Sample Transfer Arm (STA)}

The OS must hold the desired number of sample tubes as retrieved by the SFR and/or Mars 2020. The final number of tubes remains a parameter under study but the system has been designed to carry 30 tubes. The shape of the OS has been designed to be cylindrical with rounded caps to maximize the number of tubes at the lowest possible mass. Tubes would be inserted from the SFR storage rack into the OS by the STA on the lander (Fig. 3). A different technique using the same transfer arm, now equipped with a "catcher's mitt", could be used to transfer tubes still to be dropped by Perseverance.

After the OS has been filled, it would be closed and sealed (using an engineered lip seal), and the MPA cover closed for launch. The tubes need to be secured and maintained through environmental conditions through Mars launch, Earth return and Earth landing. Constraints placed on the management of the sample tubes by science include maintaining the temperature to less than $+30^{\circ} \mathrm{C}$ and magnetic field below $1 / 2 \mathrm{mT}$ (at the sample). Additionally, the OS must have sufficient albedo (assumed $>0.7$ ) to be detected in Mars orbit by visible light framing cameras on the ERO.

\section{Mars Ascent Vehicle (MAV) Concept}

The MAV performance and reliability (including launch mass and injection into orbit) was evaluated by MSFC and JPL for two propulsion technologies, a single stage to orbit hybrid (solid fuel/liquid oxidizer) or a two-stage solid (Fig. 4). The MAV would be responsible for launching the OS from the surface of Mars into a nearly circular orbit of $\sim 400 \mathrm{~km}$ altitude and 25 degree inclination.

The drivers for the MAV are its mass and size ( $\sim 3 \mathrm{~m}$ long by $0.57 \mathrm{~m}$ diameter), as both must be accommodated in the previously described lander. 


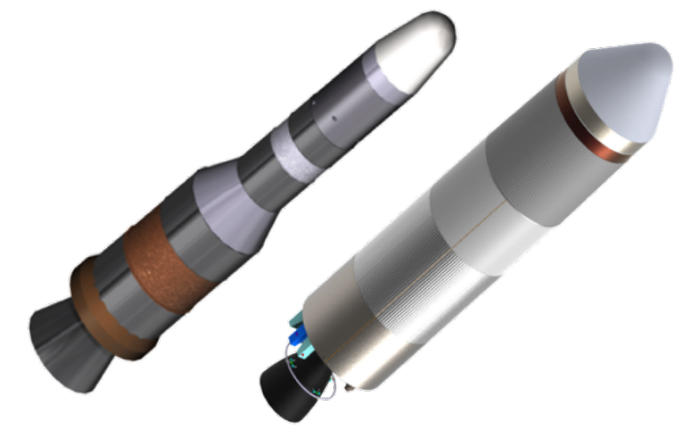

Figure 4: Solid (left) and Hybrid (right) MAV Concepts

The hybrid single-stage-to-orbit propulsion system uses a wax-based fuel and Mixed Oxides of Nitrogen (MON) oxidizer capable of being stored in the variable and low temperature conditions on Mars. [6]

The solid design would use an updated low temperature propellant previously used on the Mars Pathfinder and Mars Exploration Rovers Rocket Assisted Deceleration systems.

Based on comparable performance but the relative maturity of the solid motor technology, the solid MAV was selected as the reference baseline.

\section{Sample Fetch Rover (SFR) Concept}

ESA has selected Airbus Defense and Space UK to develop the Sample Fetch Rover (SFR) as a possible contribution to the Mars Sample Return Campaign. Airbus is currently working with JPL to define requirements and interfaces that will allow the development of a breadboard to demonstrate in a field trial the end-to-end operational concept, including the capability to traverse autonomously and pick up and store the sample tubes for transport back to the SRL.

The SFR concept is a four-wheel vehicle with ExoMars heritage for the locomotion system elements, thermal control and energy management, and autonomous navigation. SFR is also incorporating technologies developed through the ESA Mars Robotic Exploration
Preparation (MREP) Program, such as visual odometry, miniaturised avionics, and low temperature mechanisms and batteries.

The current concept would use wheels provided by NASA-GRC based on a compliant wheel technology, using a shape memory alloy tire, that has been shown to provide exceptional traversability performance.

NASA and ESA are working to establish sound interfaces between SFR and the lander, based on a top mounted SFR with NASA providing the egress system.

\section{Earth Return Orbiter (ERO) Concept}

The ESA Earth Return Orbiter (Fig. 5) functions include: 1) providing the launch and propulsion energy needed to meet the round-trip mission timeline; 2) providing the telecommunications relay function needed by the surface mission, including EDL; 3 ) executing the autonomous rendezvous and capture in Mars orbit of the OS with its samples; and 4) returning to Earth and achieving targeting for release of the EES.

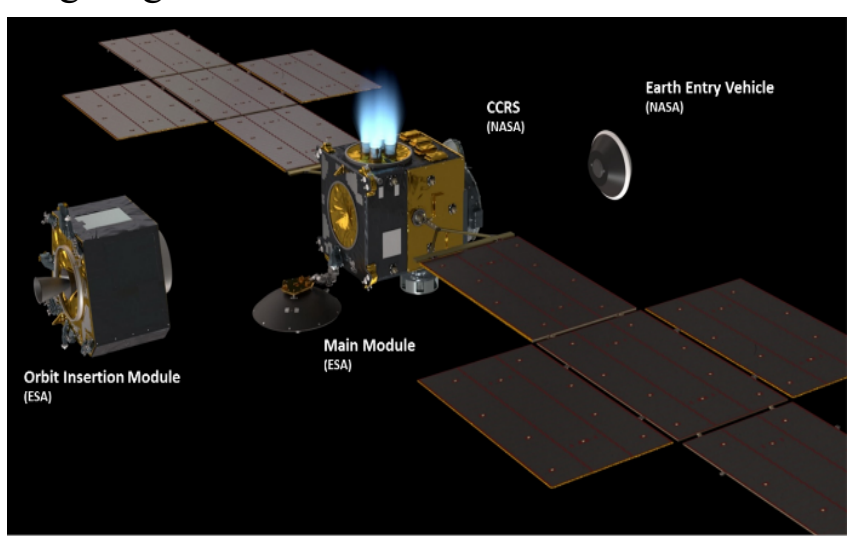

Figure 5: Earth Return Orbiter (ERO) Concept

ESA has selected the ERO contractor, Airbus Toulouse, who developed Bepi Columbo, to accommodate the NASA-provided CCRS, all to be launched on an Ariane 6.4. The ERO will use a hybrid chemical and electrical propulsion system that would provide the first roundtrip trajectory between Eath and Mars. 


\section{Capture/Containment and Return System (CCRS)}

The current CCRS concept is comprised of three major elements (Fig. 6). The Capture, Orient Module (COM) would capture, secure, orient the $\mathrm{OS}$ and then transfer it to the Containment Module (CM) to complete the first containment stage by encapsulating the OS in a primary containment vessel which by design "Breaks the Chain" of contact with Mars. A robotic arm transfers the encapsulated OS to the EES, installs a secondary containment vessel lid, and completes the closure by installing the backshell cover. To save mass, the COM/CM are jettisoned before leaving Mars orbit.

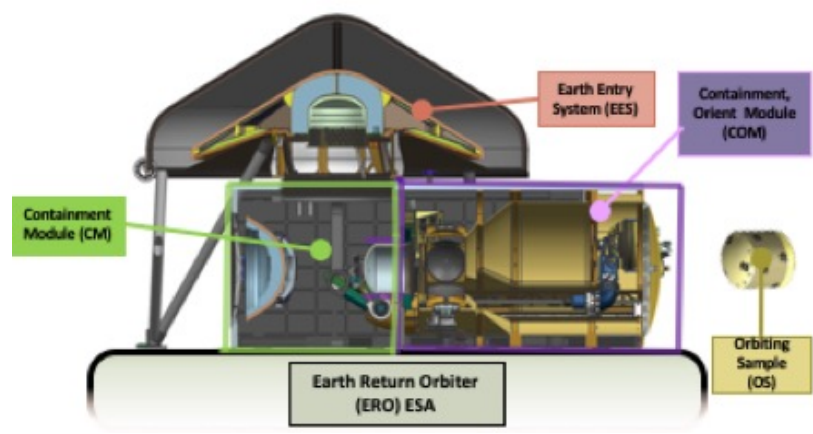

Figure 6: Capture/Containment and Return System (CCRS) Concept

The EES (shown in Fig. 6) is being designed consistent with past interplanetary Earth entry vehicles including an entry velocity of $<12$ $\mathrm{km} / \mathrm{s}$. The EES is a fully passive aerodynamic design for entry and landing without use of a parachute, driven by the high reliability needed to meet Backward Planetary Protection objectives. The EES is being designed to sustain the OS and sample tube integrity upon landing with a nominal landing load $<1300$ g's and an off-nominal hard surface landing load $<3000$ g's.

\section{Summary}

The Mars Sample Return campaign design studies at NASA and ESA are proceeding well. The MSR campaign study process has established a reference baseline that has closed on a robust campaign architecture in preparation for a MSR Campaign Concept Review in late summer 2020. NASA and ESA are working together well in an effective partnership, with roles and responsibilities clearly defined and being implemented.

\section{Acknowledgements}

The information about a potential Mars Sample Return campaign is pre-decisional and is provided for planning and discussion purposes only. Some of the work reported here was carried out at the Jet Propulsion Laboratory, California Institute of Technology, under a contract with the National Aeronautics and Space Administration.

\section{References}

[1] NRC (2011), Vision and Voyages for Planetary Science in the Decade 20132022, National Academies Press, Washington DC, ISBN: 0-309-20955-2.

[2] T. H. Zurbuchen, Mars Exploration Program, Presentation to the National Academies, 28 Aug 2017.

[3] iMOST (2018), The Potential Science and Engineering Value of Samples Delivered to Earth by Mars Sample Return, Meteoritics \& Planetary Science (https://onlinelibrary.wiley.com/doi/epdf/1 0.1111/maps.13242).

[4] H. Y. McSween, M. M. Grady et al., "Why Mars Sample Return is a Mission Campaign of Compelling Importance to Planetary Science and Exploration," White Paper for the Survey, 2020.

[5] Robert Gershman, et al. "Break-theChain Technology for Potential Mars Sample Return," IEEE Aerospace Conf., 2018, Big Sky, Montana, March, 2018.

[6] Ashley C. Karp, et al., "Technology Development for a Potential Hybrid Mars Ascent Vehicle," International Astronautical Congress, Bremen, Germany, Oct. 2018. 